T. Adachi

Nagoya Math. J.

Vol. 131 (1993), 67-74

\title{
A NOTE ON THE FØLNER CONDITION FOR AMENABILITY
}

\author{
TOSHIAKI ADACHI
}

\section{Dedicated to Professor Nobunori Ikebe for his 60th birthday}

\section{Introduction}

Let $G$ be a countably generated discrete group. $A$ right-invariant mean $\mu$ on $G$ is a bounded linear functional of the space $L^{\infty}(G)$ of bounded functions on $G$ having the property:

(1) $\inf _{x \in G} \varphi(x) \leq \mu(\varphi) \leq \sup _{x \in G} \varphi(x)$ for $\varphi \in L^{\infty}(G)$,

(2) for every $g \in G, \mu(g \cdot \varphi)=\mu(\varphi)$, where $g \cdot \varphi(x)=\varphi(x g)$.

We say that $G$ is amenable if it is equipped with a right-invariant mean. Finite groups, abelian groups, in fact, groups of subexponential growth are amenable. Solvable group are also amenable. Subgroups and quotients of amenable groups are amenable. On the other hand, free groups having two generators and over are non-amenable.

In his paper [4], Følner gives the following combinatorial characterization.

THEOREm (Følner). A countably generated discrete group $G$ is amenable if and only if, for every positive $\varepsilon$ and arbitrary finite subset $A$ of $G$, there exists a non-empty finite subset $E$ of $G$ such that

$$
\#(E \cdot a \backslash E) \leq \varepsilon \# E \text { for every } a \in A,
$$

where \#F denotes the cardinality of a set $F$.

Roughly speaking, we can choose a finite subset in an amenable group having small boundary compared with its cardinality (see $\S 1$ ). This property is quite useful in the study of the spectral theory of complete Riemannian manifolds admitting an amenable group action with compact orbit space ([1], [3]). In this note we refine this characterization from a geometric point of view, precisely, a viewpoint

Received June 4, 1992.

Supported partially by The Sumitomo Foundation. 
of the isoperimetric inequality for associated graphs, so that we can apply it in the study [2] of the heat kernel of such manifolds. In Section 2 we show that a group is amenable if and only if its isoperimetric constant is zero. In Section 3 we construct approximations realizing the isoperimetric constant for amenable groups. For more information on amenability see [6].

\section{Isoperimetric constant}

In order to state our result we introduce graph theoretic terminology. Every subset $A$ of $G$ induces a graph $\left(G, \mathscr{E}_{A}\right)$ which consists of the set of vertices $G$ and the set of edges $\mathscr{E}_{A}=\left\{(x, y) \in G \times G \mid x^{-1} y \in A \cup A^{-1}\right\}$. An $A$-path is an element of the form

$$
\begin{aligned}
& c=\left(x_{0}, x_{1}, \ldots, x_{m}\right) \in \overbrace{G \times \cdots \times G}^{m+1}, \\
& x_{\imath-1}^{-1} x_{i} \in A \cup A^{-1} \text { for } i=1, \ldots, m .
\end{aligned}
$$

In case one need to make clear the length and both ends of $c$, call it an $m$-step $A$-path joining $x_{0}$ and $x_{m}$. We say $c$ belongs to a subset $E$ of $G$ if $x_{\imath} \in E$, $i=0, \ldots, m$, and call $E$ is $A$-comnected if every two elements $g, h \in E$ can be joined by an $A$-path belonging to $E$. The notion of $A$-connectedness is equivalent to the topological connectedess in the one dimensional CW-complex $\left(G, \mathscr{E}_{A}\right)$. An $A$-connected subset $F$ of $E$ is called an $A$-connected component of $E$ if it is a maximal $A$-connected subset of $E$ i.e. if $F^{\prime}$ is $A$-connected subset of $E$ containing $F$ then $F^{\prime}=F$. Given two subsets $E$ and $F$ of $G$ we denote by $E \cdot F$ the set $\{g h \in G \mid g \in E, h \in F\}$, and by $E^{m}$ the set $\left\{g_{1} g_{2} \cdots g_{m} \in G \mid g_{1} \in E\right\}$.

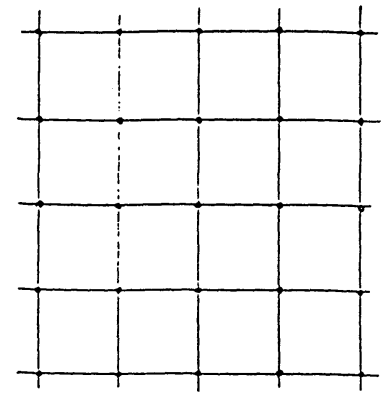

$\left(\mathbf{Z}^{2}, \mathscr{E}_{A}\right)$

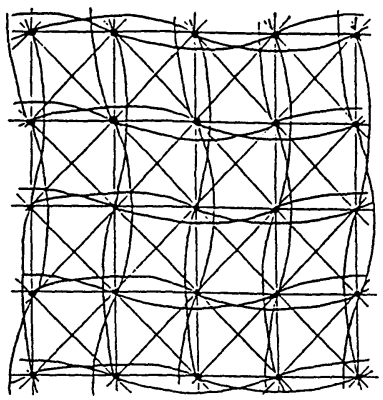

$$
\left(\mathbf{Z}^{2}, \mathscr{E}_{A^{2}}\right)
$$

where $A=\{e=(0,0),(1,0),(0,1),(-1,0),(0,-1)\}$

Figure 1 
It is clear that the Følner condition is equivalent to the condition that there is a finite subset $E$ with \# $(E \cdot A \backslash E) \leq \varepsilon \# E$ for every positive $\varepsilon$ and a finite subset $A$. We first show that in the case that $G$ is finitely generated this condition does not depend on the choice of $A$.

Propositiox 1. Let $G$ be a finitely generated group, $A$ be a finite set of generators of $G$, and $B$ be a finte subset of $G$. If there is a non-empty finite subset $E$ of $G$ with \# $(E \cdot A \backslash E) \leq \varepsilon \# E$ for every positive $\varepsilon$, then so is for $B$, i.e. there exists a non-empty finite subset $F$ with \# $(F \cdot B \backslash F) \leq \varepsilon \# F$ for every $\varepsilon$.

Proof. Choose positive $m$ so that $A^{m}$ contains $B$. Let $F$ be a finite subset satisfying \# $(F \cdot A \backslash F) \leq \varepsilon\left\{(\# A)^{m}-1\right\}^{-1}\{\# A-1\} \# F$. Since we have

$$
\begin{aligned}
F \cdot B \backslash F \subset F \cdot A^{m} \backslash F \\
\quad \subset\left(F \cdot A^{m} \backslash F \cdot A^{m-1}\right)+\left(F \cdot A^{m-1} \backslash F \cdot A^{m-2}\right)+\cdots+(F \cdot A \backslash F)
\end{aligned}
$$

and

$$
\begin{aligned}
\left(F \cdot A^{j+1} \backslash F \cdot A^{\prime}\right) & =\bigcup_{g \in A^{j}}\left(F \cdot A \cdot g \backslash F \cdot A^{\prime}\right) \\
& \subset \underset{g \in A^{\prime}}{\bigcup}(F \cdot A \cdot g \backslash F \cdot g)=\bigcup_{g \in A^{j}}(F \cdot A \backslash F) \cdot g,
\end{aligned}
$$

we get

$$
\begin{aligned}
\#(F \cdot B \backslash F) & \leq \#\left(F \cdot A^{m} \backslash F\right) \leq \sum_{j=0}^{m-1} \#\left(F \cdot A^{j+1} \backslash F \cdot A^{j}\right) \\
& \leq \sum_{j=0}^{m-1} \#\left(\bigcup_{g \in A^{\prime}}(F \cdot A \backslash F) \cdot g\right) \leq \sum_{j=0}^{m-1} \#\left(A^{j}\right) \#(F \cdot A \backslash F) \\
& \leq \sum_{j=0}^{m-1}(\# A)^{j} \varepsilon\left\{(\# A)^{m}-1\right\}^{-1}\{\# A-1\} \# F \leq \varepsilon \# F .
\end{aligned}
$$

We now consider a geometric meaning of \# $(E \cdot A \backslash E) \leq \varepsilon \# E$. The set $\{g\} \cdot\left\{A \cup A^{-1}\right)$ is the nearest neighbourhood of $g \in G$, that is the set of points which can be joined by a 1 -step $A$-path. We therefore call the set $E \cdot\left(A \cup A^{-1}\right) \backslash E$ the exterior $A$-boundary of $E$. (See Figure 2.) In the case that $A$ is finite and symmetric, i.e. $A^{-1}=A$, the inequality \# $(E \cdot A \backslash E) \leq \varepsilon \# E$ states a relationship between the volume of $E$ and the volume of its exterior $A$-boundary, which we may call isoperimetric inequality. In geometry it is natural to restrict $E$ to be "connected". It should be noted that there exists a subset of $G$ which is not $A$-connected but whose exterior $A$-boundary is connected. 


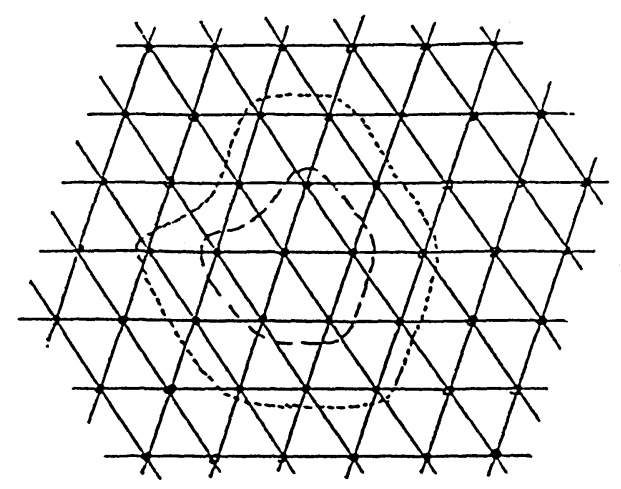

Figure 2

THEOREM 2. A countably generated discrete group $G$ is amenable if and only if, for every positive $\varepsilon$ and every finite subset $A$ of $G$, there exists a non-empty $A$-connected finite subset $E$ of $G$ such that

$$
\#(E \cdot a \backslash E) \leq \varepsilon \# E \text { for every } a \in A .
$$

In other words, $G$ is amenable if and only if the isoperimetric constant

$$
\iota_{A}(G)=\inf \left\{\frac{\#(E \cdot A \backslash E)}{\# E} \mid E \text { is an } A \text {-connected finite subset }\right\}
$$

is equal to zero for every finite subset $A$.

Proof. If the condition holds then $G$ is amenable by the Følner's theorem. Conversely, suppose $G$ is amenable. Since $\left(A \cup A^{-1} \cup\left\{1_{G}\right\}\right)$-connectedness is equivalent to $A$-connectedness it is enough to look for a connected set for symmetric $A$ which contains the unit element $1_{G}$. By the Følner condition there is a finite subset $F$ satisfying \# $(F \cdot A \backslash F) \leq \varepsilon(\# A)^{-1} \# F$. Let $F=F_{1}+F_{2}+\cdots+F_{l}$ be the decomposition into $A^{2}$-connected components. Because $F_{\imath} \cdot A \cap F_{i^{\prime}}=\phi$ and $F_{i} \cdot A \cap F_{i^{\prime}} \cdot A=\phi$ for $i \neq i^{\prime}$, we have

$$
F \cdot A \backslash F=\bigcup_{i=1}^{l}\left(F_{i} \cdot A \backslash F\right)=\sum_{i=1}^{l}\left(F_{i} \cdot A \backslash F_{\imath}\right),
$$

hence \# $\left(F_{i_{0}} \cdot A \backslash F_{i_{0}}\right) \leq \varepsilon(\# A)^{-1} \# F_{i_{0}}$ for some $i_{0}$.

Put $E=F_{i_{0}} \cdot A$. We see that

$$
E \cdot A \backslash E=\bigcup_{a \in A}\left(F_{\imath_{0}} \cdot A \cdot a \backslash F_{\imath_{0}} \cdot A\right) \subset \bigcup_{a \in A}\left(F_{i_{0}} \cdot A \backslash F_{i_{0}}\right) \cdot a,
$$

and 


$$
\#(E \cdot A \backslash E) \leq \sum_{a \in A} \#\left(\left(F_{i_{0}} \cdot A \backslash F_{i_{0}}\right) \cdot a\right) \leq \varepsilon \# F_{i_{0}} \leq \varepsilon \# E
$$

We now show that $E$ is $A$-connected. Choose arbitrary points $f a$ and $f^{\prime} a^{\prime}$ in $E$, where $f, f^{\prime} \in F_{\imath_{0}}$ and $a, a^{\prime} \in A$. There is an $A^{2}$-path $c$ joining $f$ and $f^{\prime}$ :

$$
c=\left(x_{0}, x_{1}, \ldots, x_{m}\right), x_{0}=f, x_{m}=f^{\prime}, x_{j} \in F_{i_{0}} \text {, and } x_{j-1}^{-1} x_{j} \in A^{2} \text {. Put } y_{0}=x_{0} \text {. }
$$

For $j=1, \ldots, m-1$, we set

$$
\begin{aligned}
y_{2 j-1}= & y_{2 j}=x_{j}, \text { if } x_{j-1}^{-1} x_{j} \in A, \\
y_{2 j-1}= & x_{j-1} a_{j} \text { and } y_{2 j}=y_{2 j-1} a_{j}^{\prime}=x_{\jmath}, \\
& \text { if } x_{j-1}^{-1} x_{j}=a_{j} a_{j}^{\prime} \in A^{2} \backslash A\left(a_{j}, a_{j}^{\prime} \in A\right) .
\end{aligned}
$$

Finally we put $y_{2 m-1}=x_{m}$. Then

$$
\left(f a, y_{0}, y_{1}, \ldots, y_{2 m-1}, f^{\prime} a^{\prime}\right)
$$

is an $A$-path belonging to $E$, hence $E$ is $A$-connected .

By using Proposition 1, we have the following important remark.

Remark. In the case that $G$ is finitely generated, if $\iota_{A}(G)=0$ for some generator set $A$ then $\iota_{B}(G)=0$ for every finite subset $B$. Hence in order to show that $G$ is amenable we may only check this isoperimetric condition for some generator set.

\section{Connected summing sequences}

In this section we show that the set $E$ in Theorem 2 can be chosen arbitrary large. Let $A$ be a finite subset of $G$. A sequence $\left\{E_{j}\right\}_{j=1}^{\infty}$ of non-empty $A$-connected finite subsets of $G$ is called an $m$-thick $A$-summing sequence for $G$ if the following conditions hold:

(1) $E_{j} \subset E_{\jmath+1}$ and $G=\bigcup_{j=1}^{\infty} E_{\jmath}$,

(2) \# $\left(E_{j} \cdot A^{m} \backslash E_{j}\right) / \# E_{j} \rightarrow 0$ as $j \rightarrow \infty$.

If such a sequence exists for $m=1, G$ is finitely generated and amenable by Theorem 2. One of the aim of this section is to show the converse. Before do that we consider a geometric meaning of the condition (2) when $A$ is symmetric and contains the unit element. We have a natural distance function on $G$; the distance $d_{A}(x, y)$ between $x$ and $y(x, y \in G)$ is given by the infimum of length of 
$A$-paths joining these elements. Using this distance we can express the set $E \cdot A^{m}$ as $\left\{x \in G \mid d_{A}(x, E) \leq m\right\}$. So \# $\left(E \cdot A^{m} \backslash E\right)$ means the volume of the "thick" boundary of $E$. The second aim of this section is to show that a finitely generated group $G$ is amenable if and only if the isoperimetric constants corresponding to thick boundaries are 0 .

By a slight modification of the proof of Theorem 2, we have

Proposition 3. A countably generated discrete group $G$ is amenable if and only if, for every positive $\varepsilon$, every positive integer $m$, and arbitrary finite subset $A$, there exists a non-empty $A$-connected finite subset $E$ of $G$ satisfying \# $\left(E \cdot A^{m} \backslash E\right) \leq \varepsilon \# E$.

Using this inductively, we get

THeorem 4. A group $G$ with a finite set $A$ of generators is amenable if and only if, for every positive integer $m$, there exists an $m$-thick $A$-summing sequence.

Proof. If there exist summing sequences then $G$ is amenable by Følner's theorem. Conversely, suppose $G$ is amenable. We may suppose that $A$ containing the unit element. Let $E_{1}$ be a finite $A$-connected subset such that $1_{G} \in E_{1}$ and $\#\left(E_{1} \cdot A^{m} \backslash E_{1}\right) \leq \# E_{1}$. If we have a finite $A$-connected subset $E_{\jmath-1}$ satisfying

$$
1_{G} \in E_{j-1} \text { and } \#\left(E_{\jmath-1} \cdot A^{m} \backslash E_{\jmath-1}\right) \leq \frac{1}{j-1} \# E_{j-1} \text {, }
$$

we choose an $A$-connected finite set $E_{j}^{\prime}$ so that

$$
1_{G} \in E_{j}^{\prime} \text { and } \#\left(E_{j}^{\prime} \cdot A^{m} \backslash E_{j}^{\prime}\right) \leq \frac{1}{j} \# E_{j}^{\prime}\left\{\# E_{j-1}+(\# A)^{\prime}\right\}^{-1} .
$$

Put

$$
E_{j}=\underset{g \in E_{j-1} \cup A^{j}}{\bigcup} g \cdot E_{j}^{\prime}
$$

Since $E_{j-1}$ is $A$-connected and $1_{G} \in E_{j-1} \cap A^{j}$, the set $E_{j-1} \cup A^{j}$ is $A$-connected. Hence $E$, is $A$-connected, as in the above definition $g$ runs over $E_{j-1} \cup A^{j}$ and $g \in g \cdot E_{j-1}$, Moreover we have $E_{j-1} \subset E_{\jmath}$, and

$$
E_{j} \cdot A^{m} \backslash E_{j}=\underset{g \in E_{j-1} \cup A^{j}}{U}\left(g \cdot E_{j}^{\prime} \cdot A^{m} \backslash E_{j}\right) \subset \underset{g \in E_{j-1} \cup A^{j}}{\bigcup} g \cdot\left(E_{j}^{\prime} \cdot A^{m} \backslash E_{j}^{\prime}\right),
$$

so 


$$
\#\left(E_{j} \cdot A^{m} \backslash E_{\jmath}\right) \leq \#\left(E_{\jmath-1} \cup A^{\jmath}\right) \#\left(E_{j}^{\prime} \cdot A^{m} \backslash E_{\jmath}^{\prime}\right) \leq \frac{1}{j} \# E_{\jmath}^{\prime} \leq \frac{1}{j} \# E_{\jmath} .
$$

Since $A$ generates $G$, we have $\cup_{j=1}^{\infty} E_{j} \supset \cup_{j=1}^{\infty} A^{j}=G$, therefore $\left\{E_{j}\right\}_{j=1}^{\infty}$ is an $m$-thick $A$-summing sequence of $G$.

We should point out that, by a diagonal argument, a finitely generated group $G$ is amenable if and only if, for arbitrary finite set $A$ there is a sequence $\left\{E_{j}\right\}_{j=1}^{\infty}$ of finite subsets which is an $m$-thick $A$-summing sequence for every $m$.

When $G$ is of subexponential growth, we have a canonical connected summing sequence which consists of metric balls.

Exalyle. Suppose $G=\mathbf{Z}^{n}$, a free abelian group with generators $a_{1}, \ldots, a_{n}$. Putting $A=\left\{1_{G}, a_{1}, a_{1}^{-1}, \ldots, a_{n}, a_{n}^{-1}\right\}$, we put $E_{j}=A^{j^{2}}=\left\{x \in G \mid d_{A}\left(x, 1_{G}\right)\right.$ $\left.\leq j^{2}\right\}$. Then $\left\{E_{j}\right\}_{j=1}^{\infty}$ is an $m$-thick $A$-summing sequence for every $m$.

Propositiox 5. Let $G$ be a finitely generated group of subexponential growth and $A$ be a symmetric finite set of generators containing the unit element. Then there exists a sequence $\left\{r_{j}\right\}_{j=1}^{\infty}$ of positive integers such that the metric balls $A^{r_{j}}=\left\{x \in G \mid d_{A}\left(x, 1_{G}\right)\right.$ $\leq r_{j}$ \} make up an $m$-thick $A$-summing sequence for every $m$.

Proof.. We first show that, for every positive $\delta$ and every positive integers $k$ and $r$, there is a positive integer $M$ satisfying $M>r$ and $\#\left(A^{M+k}\right) \leq e^{\delta} \#\left(A^{M}\right)$, here $e$ denotes the base of natural logarithm. If it is not true, we have $\delta, k$ and $r$ such that $e^{\delta} \#\left(A^{M}\right)<\#\left(A^{M+k}\right)$ for every $M \geq r$. Let $N=N(M)$ be the integer with $r+N k \leq M<r+(N+1) k$. We have

$$
e^{N \delta} \#\left(A^{r}\right) \leq e^{N \delta} \#\left(A^{M-N k}\right) \leq e^{(N-1) \delta} \#\left(A^{M-(N-1) k}\right) \leq \cdots \leq \#\left(A^{M}\right) .
$$

Since $N \geq(M-r) / k-1$, we get

$$
\left(\frac{M-r}{k}-1\right) \delta+\log \#\left(A^{r}\right) \leq \log \#\left(A^{M}\right), \text { for } M \geq r,
$$

and $G$ is of exponential growth; $\lim _{M \rightarrow \infty} M^{-1} \log \#\left(A^{M}\right) \geq \delta / k>0$, which is a contradiction.

Let $\left\{\delta_{j}\right\}_{j=1}^{\infty}$ be a sequence of positive numbers satisfying $\lim \delta_{j}=0$. By the above argument we have a positive integer $r_{1}$ with $\#\left(A^{r_{1}+1}\right) \leq e^{\delta_{1}} \#\left(A^{r_{1}}\right)$. Inductively we can choose positive integers $r_{\text {, so that }} r_{j}>r_{j-1}$ and $\#\left(A^{r,+j}\right) \leq e^{\delta_{j}}$ $\#\left(A^{r_{j}}\right)$. Since $A$ contains the unit element, $A^{M} \subset A^{N}$ if $M \leq N$, therefore we have for $j \geq m$ 


$$
\frac{\#\left(A^{r_{j}} \cdot A^{m} \backslash A^{r_{j}}\right)}{\#\left(A^{r_{j}}\right)}=\frac{\#\left(A^{r_{j}+m}\right)-\#\left(A^{r_{j}}\right)}{\#\left(A^{r_{j}}\right)} \leq \frac{\#\left(A^{r_{j}+j}\right)-\#\left(A^{r_{j}}\right)}{\#\left(A^{r_{j}}\right)} \leq e^{\delta_{j}}-1 .
$$

Hence $\left\{A^{r_{j}}\right\}_{j=1}^{\infty}$ is an $m$-thick $A$-summing sequence.

\section{REFERENCES}

[1] T. Adachi, Spherical mean and the fundamental group, Canad. Math, Bull., 34 (1991), 3-11.

[2] T. Adachi and T. Sunada, Integrated density of states for the Laplacian, to appear in Comment. Math. Helv.

[3] R. Brooks, The fundamental group and the spectrum of the Laplacian, Comment. Math. Helv., 56 (1981), 581-598.

[4] E. Følner, On groups with full Banach mean value. Math. Scand., 3 (1955), $243-254$.

[5] F. P. Greenleaf, Invariant Means on Topological Groups and Their Applications, Van Nostrand, New York 1969.

[6] A. L. Paterson, Amenability, Methematical Surveys and Monographs 29, A.M.S 1988.

Department of Mathematics

Nagoya Institute of Technology

Showa-ku, Nagoya 466, Japan

e-mail address: d43019a@nucc.cc.nagoya-u.ac.jp 